\title{
FINITE GROUPS AS ISOMETRY GROUPS
}

\author{
BY \\ D. ASIMOV
}

\begin{abstract}
We show that given any finite group $G$ of cardinality $k+1$, there is a Riemannian sphere $S^{k-1}$ (imbeddable isometrically as a hypersurface in $\mathrm{R}^{k}$ ) such that its full isometry group is isomorphic to $G$. We also show the existence of a finite metric space of cardinality $k(k+1)$ whose full isometry group is isomorphic to $G$.
\end{abstract}

Let $G$ be a finite group of $k+1$ elements $\left\{1, g_{1}, \ldots, g_{k}\right\}$.

THEOREM. There exists a Riemannian metric on the sphere $S^{k-1}$ such that the isometry group is isomorphic to $G$.

Proof. Label the $k+1$ vertices of a regular $k$-simplex $\Delta_{k}$ by the names $1, g_{1}, \ldots, g_{k}$ of the elements of $G$. Assume $\Delta_{k}$ to be inscribed in a standard $S^{k-1}$ sitting in $\mathrm{R}^{k}$ as usual. $T_{y}\left(S^{k-1}\right)$ denotes the tangent space at $y$.

Now in $T_{1}\left(S^{k-1}\right)$ pick an orthonormal frame $\left(v_{1}, \ldots, v_{k-1}\right)$. Pick $\epsilon>$ 0 small and let

$$
w_{i}=\epsilon\left(1+(i-1) / 4 k^{2}\right) v_{i}, \quad 1 \leqslant i \leqslant k-1
$$

Let

$$
Q=\left\{\exp _{1}\left(w_{i}\right) \mid 1 \leqslant i \leqslant k-1\right\} \cup\left\{\exp _{1}(0)\right\} \cup\left\{w_{1} / 10\right\} .
$$

$\exp _{1}$ is the exponential map $\exp _{1}: T_{1}\left(S^{k-1}\right) \rightarrow S^{k-1}$.

Think of $G$ as acting on $S^{k-1}$ by the isometries induced from the permutation representation on the vertices of $\Delta_{k}$. Let $X=\{g Q \mid g \in G\}$.

Proposition. With the induced metric from $\mathbf{R}^{k}$, the metric space $X$ has its group of isometries isomorphic to $G$.

Proof. Clearly $G$ acts as a group of isometries of $X$, since $X=$ $h\{g Q \mid g \in G\}=\{h g Q \mid g \in G\}=\{g Q \mid g \in G\}=X$.

Conversely, any isometry of $X$ must take the point 1 to some point $g$, since the points $g$ are characterized by being the only points in $X$ having their

Received by the editors May 8, 1975.

AMS (MOS) subject classifications (1970). Primary 53C20, 54E40, 20C99; Secondary 20D99, 54E35, 53C40.

Key words and phrases. Group, isometry, Riemannian, manifold, the metric space. 
two nearest neighbors at distance of $\epsilon / 10$ and $\epsilon$ respectively. Once we know that $1 \mapsto g$, the configuration $g Q$ determines the image of the frame $\left(w_{1}, \ldots\right.$, $\left.w_{k-1}\right)$ at 1 , and hence determines the unique isometry of $X$ defined by the element $g \in G$. Of course $\epsilon$ must be chosen small enough so that the configurations $g Q, g \in G$ do not "interfere" with one another.

Now we add bumps to $S^{k-1}$ at the points of $X$ using scalar multiplication in $\mathbf{R}^{k}$. Let

$$
\delta=(1 / 3) \min \left\{\text { dist }_{s^{k-1}}(x, y) \mid x, y \in X\right\} .
$$

Let $f:[0, \delta] \rightarrow \mathbf{R}$ be a smooth function satisfying

(a) $f(s)=100,0 \leqslant s \leqslant \delta / 2$,

(b) $f(\delta)=1 ; f^{(k)}(\delta)=0, k=1,2, \ldots$,

(c) $f^{(k)}(\delta / 2)=0, k=1,2, \ldots$, and

(d) $f^{\prime}(s)<0$ if $\delta / 2<s<\delta$.

Now for each point $x \in X$ we remove the disk $\exp _{x}\left(D_{\delta}\right)$ from $S^{k-1}$ and replace it by the point set $B_{x}=\left\{(f(|v|)) \exp _{x}(v) \mid v \in D_{\delta}\right\}$, where $D_{\delta}$ is the ( $\delta$ )-disk about the origin of $T_{x}\left(S^{k-1}\right)$. Clearly the set $S^{k-1}-\bigcup_{x \in X} \exp _{x}\left(D_{\delta}\right)$ $\cup \bigcup_{x \in X} B_{x}$ is a smooth $S^{k-1}$ imbedded in $\mathbf{R}^{k}$. We give it the induced Riemannian metric from $\mathbf{R}^{k}$ and denote it by $M$.

CLAIM: $\operatorname{Isom}(M) \approx G$.

Proof. First we notice that the points of $100 \cdot X \subset M$ must be taken to themselves by any isometry $I$ of $M$, by the choice of the function $f$. Clearly the same arguments above for $X$ hold for $100 \cdot X$, hence the isometry $I: M \rightarrow M$ restricted to $100 \cdot X$ comes from the action of $G$.

Let us now consider the "bump" $B_{1}$ above the point 1 . Let us define for $r \geqslant 0, S_{r}=\left\{f(r) \cdot \exp _{1}(v)|| v \mid=r, v \in T_{1}\left(S^{k-1}\right)\right\}$. In other words, $S_{r}$ is the $(k-2)$-sphere of $B_{1}$ lying above the $(k-2)$-sphere about 1 of radius $r$, for $0<r \leqslant \delta$, and for $r=0$ we set $S_{0}=p$, the peak point of $B_{1}$.

Now it is easy to show that the orthogonal trajectories of the $S_{r}$ 's are geodesics of $M$ and as such must be preserved under any isometry taking $p$ to $p$.

Thus any isometry $I$ of $M$ which takes $p$ to $p$ (and which must thus leave all points of $100 \cdot X$ fixed) must be a "rotation" on all of $B_{1}$, determined by $I \mid \partial B_{1}$, carrying each $S_{r}$ into itself by the "same" element of $O(k-2)$. Similarly, this I must rotate each bump $B_{x}, x \in X$.

Also this rotation must extend past the boundary of the bumps for some ways, so we can easily extend $I \mid\left(M-\cup_{x} B_{x}\right)$ to an isometry $\widetilde{I}$ of $S^{k-1}$ to itself, by simply "coning" I over $\exp _{x}\left(D_{\delta}\right), x \in X$. Clearly we will have $\widetilde{I}(x)=$ $x$ for $x \in X$, and it follows easily that $\widetilde{I}: S^{k-1} \rightarrow S^{k-1}$ is the identity. Hence $I: M \rightarrow M$ must have been the identity. 
Now it is clear that for each $g \in G$ there is one isometry of $M$ determined by the antion of $g$ on $S^{k-1}$, extended to $R^{k}$, restricted to $M$. Now if there is another isometry $I: M \rightarrow M$ such that $I|X=g| X$, then $I \circ g^{-1}: M \rightarrow M$ must leave points of $X$ fixed, so by the above discussion must be the identity. This establishes $\operatorname{Isom}(M) \approx G$.

COROllary. Any finite group $G$ is isomorphic to the (full) isometry group of a finite subset $X_{G}$ of euclidean space. If $\operatorname{card}(G)=k$ then the $X_{G}$ can be found with $\operatorname{card}\left(X_{G}\right)=k^{2}-k$ in euclidean space of dimension $k-1$.

Proof. Simply take $X_{G}=X$ in the proof of the Theorem, and count (noting that we initially took $\operatorname{card}(G)=k+1$ ).

REMARK. Further considerations can very likely reduce the necessary cardinality for $X_{G}$ to $k(k-3)$. The various numbers

$$
\begin{aligned}
& d=\min \{\operatorname{card}(X) \mid G \approx \operatorname{Isom}(X)\} \text { and } \\
& e=\min \{N \mid G \text { has a faithful representation into } O(N)\}
\end{aligned}
$$

seem to be interesting invariants of a finite group $G$.

SCHOOL OF MATHEMATICS, UNIVERSITY OF MINNESOTA, MINNEAPOLIS, MINNESOTA 55455

Current address: School of Mathematics, The Institute for Advanced Study, Princeton,
ersey 08540 New Jersey 08540 